PPPL-3471

PPPL-3471

UC-70

Fusion Ignition Research Experiment

by

Dale Meade

July 2000

$\int D \int D \int D S \int S \begin{aligned} & \text { PAINCETON } \\ & \text { PLABMA PHYSICS } \\ & \text { LABOAATOAY }\end{aligned}$

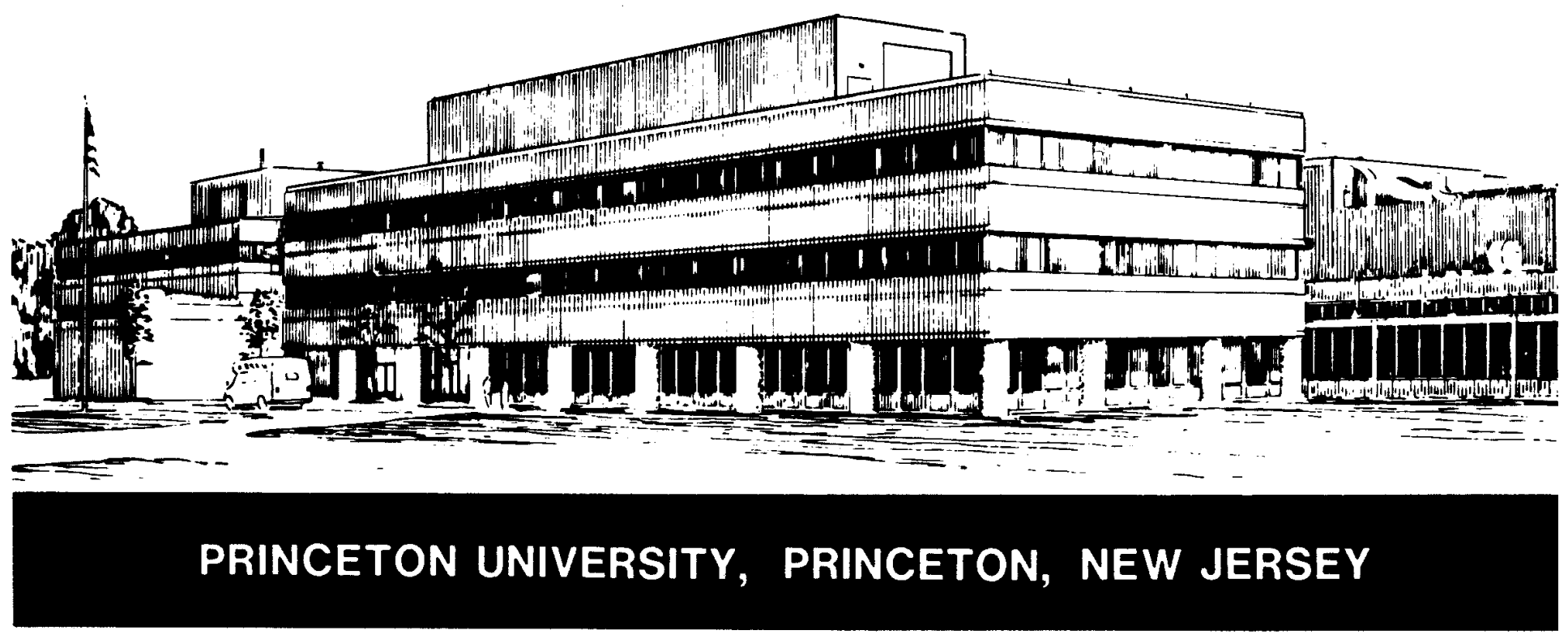




\section{PPPL Reports Disclaimer}

This report was prepared as an account of work sponsored by an agency of the United States Government. Neither the United States Government nor any agency thereof, nor any of their employees, makes any warranty, express or implied, or assumes any legal liability or responsibility for the accuracy, completeness, or usefulness of any information, apparatus, product, or process disclosed, or represents that its use would not infringe privately owned rights. Reference herein to any specific commercial product, process, or service by trade name, trademark, manufacturer, or otherwise, does not necessarily constitute or imply its endorsement, recommendation, or favoring by the United States Government or any agency thereof. The views and opinions of authors expressed herein do not necessarily state or reflect those of the United States Government or any agency thereof.

\section{Availability}

This report is posted on the U.S. Department of Energy's Princeton Plasma Physics Laboratory Publications and Reports web site in Calendar Year 2000. The home page for PPPL Reports and Publications is: http://www.pppl.gov/pub_report/

DOE and DOE Contractors can obtain copies of this report from:

U.S. Department of Energy

Office of Scientific and Technical Information

DOE Technical Information Services (DTIS)

P.O. Box 62

Oak Ridge, TN 37831

Telephone: (865) 576-8401

Fax: (865) 576-5728

Email: reports@adonis.osti.gov

This report is available to the general public from:

National Technical Information Service

U.S. Department of Commerce

5285 Port Royal Road

Springfield, VA 22161

Telephone: $1-800-553-6847$ or

(703) $605-6000$

Fax: (703) 321-8547

Internet: http://www.ntis.gov/ordering.htm 


\title{
Fusion Ignition Research Experiment
}

\author{
Dale Meade \\ Princeton Plasma Physics Laboratory
}

\begin{abstract}
Understanding the properties of high gain (alpha-dominated) fusion plasmas in an advanced toroidal configuration is the largest remaining open issue that must be addressed to provide the scientific foundation for an attractive magnetic fusion reactor. The critical parts of this science can be obtained in a compact high field tokamak which is also likely to provide the fastest and least expensive path to understanding alpha-dominated plasmas in advanced toroidal systems.
\end{abstract}

\section{Technical Challenges for Major Next Steps in Magnetic Fusion Energy (MFE)}

Tokamak experiments have achieved plasma performance $(\mathrm{n} \tau \mathrm{T})$ within an order of magnitude of that required for a high gain burn in deuterium-tritium (D-T) as illustrated in Fig. 1. The initial burning plasma experiments on TFTR and JET were successfully carried out with D-T fuel where the alpha particle effects were perturbations on the plasma dynamics. The exploration and understanding of reactor plasma dynamics where alpha particles dominate the plasma dynamics is the crucial next step needed to provide the science foundation for the Energy Development Phase of magnetic fusion. The tokamak is the only magnetic configuration capable of accessing the high gain (alpha-dominated) burning plasma regime within the next decade to provide generic knowledge on magnetically confined burning plasmas.

The functional fusion plasma requirements for major next steps in MFE can be described as:

Burning Plasma Physics - The achievement and understanding of self-heated plasmas with high gain that have characteristics similar to those expected in a fusion energy source, and

Advanced Toroidal Physics - The achievement and understanding of sustained self-heated plasmas with characteristics (steady-state or high duty factor pulsed systems) similar to those expected in a competitive fusion system

These requirements lead naturally to a set of Stepping Stones as illustrated in Fig. 2. The plasma performance and duration to address these issues are shown schematically in terms of the natural time scale for the important plasma processes.

This paper describes the capability of compact high-field tokamaks to address alpha-dominated burning plasma physics, long-pulse advanced-toroidal physics and fusion technology as part of a Modular Pathway to Magnetic Fusion Energy. The conclusion is that a compact high field tokamak utilizing LN cooled copper-alloy coils has the capability to address a major portion of both the Burning Plasma Experiment Step, the Advanced Toroidal Experiment Step and also has significant capability to integrate burning plasma physics with advanced toroidal physics. The size of the compact tokamak was constrained with the goal to achieve the most important physics goals at a construction cost of $\angle \$ 1 \mathrm{~B}$, while allowing the opportunity for later facility upgrades during the operating phase.

\section{The Next Frontier in MFE Research - Attainment, exploration, optimization and understanding of alpha-dominated burning plasmas.}

The attainment and control of a high Q plasma dominated by alpha heating is the single most important requirement for any approach to fusion power. Fusion gains $Q \geq 20$ are needed for an economical magnetic fusion reactor that is sustained at near steady-state conditions; at this $Q$ value 
the alpha particles dominate the plasma dynamics, providing $\geq 80 \%$ of the plasma heating. The goal for the Next Step in Magnetic Fusion is to access sustained alpha-dominated plasmas with alpha heating fractions more than an order of magnitude higher than present experiments.

Present day tokamaks improve plasma performance by controlling density, pressure, plasma current and transport profiles. The advanced tokamak, advanced stellarator and the spherical torus all plan to have the bootstrap current, generated by gradients in the pressure profile, produce a large fraction of the current needed to define the stabilizing magnetic field. Since the alpha heating profile is directly linked to the pressure profile, this process becomes very non-linear in alpha dominated plasmas required for fusion reactors based on advanced tokamak. This coupling of advanced tokamak confinement and MHD stability physics with alpha-dominated plasmas is a key generic issue for the development of attractive toroidal magnetic reactors.

\section{Alpha Heating Fraction, the metric for alpha-dominated burning plasmas}

The alpha heating strength can be expressed in terms of $\mathrm{f}_{\alpha}=\mathrm{P}_{\alpha} /\left(\mathrm{P}_{\alpha}+\mathrm{P}_{\text {ext }}\right)$ where $\mathrm{P}_{\alpha}$ is the alpha heating and $P_{\text {ext }}$ is the externally applied heating. The fraction of alpha heating, $\mathrm{f}_{\alpha}$, is plotted in Fig. 3 in terms of the ratio $n \tau_{\mathrm{E}} / n \tau_{\mathrm{E}}(\mathrm{Q}=\infty)$. D-T experiments on TFTR and JET have measured small temperature increases in agreement with the expected alpha particle heating. The sustained $\mathrm{D}$-T discharges on TFTR and JET had $\mathrm{Q} \approx 0.2$ for $\sim 10$ energy confinement times with the alpha particles providing only $4 \%$ of the overall plasma heating. The vision of an MFE fusion reactor is ARIES-RS, an advanced tokamak with a $\mathrm{Q}=25$ has $\mathrm{f}_{\alpha}=0.83$. The investigation of an alpha dominated plasma can begin at $\mathrm{f}_{\alpha}=0.5$, and plasmas with $\mathrm{f}_{\alpha}=0.66$ to 0.83 would match the dynamics expected in the MFE reactor regime. Note, small reductions in confinement produce only small changes in the alpha heating fraction, while $Q$ can change by a large amount especially in the high Q regime.

Fusion Ignition Research Experiment (FIRE) - A Next Step Option for MFE.

The mission of FIRE is to attain, explore, understand and optimize alpha-dominated plasmas that will provide knowledge needed for the design of attractive MFE systems. The guiding design philosophy is that FIRE must have the capability and flexibility of studying and resolving the physics issues relevant to the design of a subsequent advanced integrated fusion facility. A major consideration is to accomplish this physics mission at the lowest possible cost, with a target cost $<\$ 1 B$. The device investigated is similar in scale to the Compact Ignition Tokamak (CIT) studied in 1989. The plasma configuration was drawn from the advanced tokamak design work done for the Tokamak Physics Experiment (TPX) and is $\mathrm{a} \approx 1 / 3$ scale model of the Advanced Reactor Innovation Evaluation Study (ARIES-RS) based on a reversed shear advanced tokamak.

\section{FIRE Physics Objectives}

The physics objectives of FIRE developed to satisfy the mission are to:

1. Determine and understand the conditions required to achieve alpha-dominated plasmas:

- Energy confinement scaling with alpha-dominated heating

- $\beta$-limits with alpha-dominated heating

- Density limit scaling with alpha-dominated heating

2. Explore the dynamics of alpha-dominated plasmas using active control techniques.

3. Sustain alpha-dominated plasmas with high-power-density exhaust of plasma particles and energy and alpha ash exhaust in regimes suitable for future toroidal reactors.

4. Explore and understand alpha-dominated plasmas in advanced operating modes and configurations that have the potential to lead to attractive fusion applications. 
5. Understand the effects of fast alpha particles on plasma behavior in relevant regimes.

\section{Choice of FIRE Plasma Performance Requirements.}

FIRE is a physics experiment to extend the frontiers of fusion plasma physics into previously unexplored parameter space using advanced capabilities and flexibility for later upgrades; it is not a demonstration of the scientific and technological feasibility of magnetic fusion. The strategy for the FIRE program is to have a first stage of burning plasma experiments aimed at accessing the alpha-dominated regime with a minimum $\mathrm{f}_{\alpha}$ of $\geq 0.5$ using projections from the middle of the present tokamak performance database. This would provide a test bed where alpha heating effects are easily observable, and the plasma dynamics could still be controlled externally. This capability is the natural starting point for an experimental campaign to study alpha-dominated plasmas and would be sufficient to accomplish a significant fraction of the stated objectives. The goal for the second stage of burning plasma experiments is to achieve strongly alpha-dominated plasmas with $\mathrm{f}_{\alpha}=0.66$ to 0.83 . This level of performance is projected from the best results of the present tokamak performance database, or by a modest $20 \%$ improvement in confinement from employing advanced tokamak physics that is expected to be developed by the ongoing base tokamak program over the next 5 years.

The pulse length, or the burn time, is a very important consideration for any burning plasma experiment. The physics time scales of interest (with typical values for FIRE plasmas) are:

- $\tau_{\alpha \mathrm{\alpha}}$, the time needed for the alpha particle to transfer its energy to the plasma $(\sim 0.1 \mathrm{~s})$

- $\tau_{\mathrm{E}}$, the plasma energy confinement time $(\sim 0.6 \mathrm{~s})$

- $\tau_{\mathrm{He}}$, the confinement time of alpha ash, slowed down alpha particles $\left(\sim 5 \tau_{\mathrm{E}} \sim 3 \mathrm{~s}\right)$

- $\tau_{\mathrm{cr}}$, the time for the plasma current profile to redistribute after a perturbation $(\sim 13 \mathrm{~s})$

The characteristic time scales for plasma phenomena in FIRE plasmas are significantly shorter than the corresponding time scales on ITER-RC due to the smaller size, higher density and somewhat lower plasma temperature as shown in Table I.

Table I. Characteristic time scales for plasma phenomena in FIRE and ITER-RC.

\begin{tabular}{|l|c|c|c|c|}
\hline For $\mathrm{Q} \approx 10$ & $\tau_{\mathrm{E}}(\mathrm{s})$ & $\tau_{\mathrm{He}}(\mathrm{s})$ & $\tau_{\mathrm{cr}}(\mathrm{s})$ & $\tau_{\text {burn }}(\mathrm{s})$ \\
\hline FIRE & 0.6 & 3 & $\sim 13$ & 15 \\
\hline ITER-RC & 2.5 & 12.5 & $\sim 200$ & 450 \\
\hline
\end{tabular}

A FIRE plasma with a burn time of $15 \mathrm{~s}\left(\sim 25 \tau_{\mathrm{E}}\right)$ would allow the pressure profile to come into equilibrium with alpha heating and allow the alpha ash to accumulate for $\sim 5 \tau_{\mathrm{He}}$. This pulse length would be sufficient to address Physics Objectives 1, 2, 3, and 5. A significant part of Physics Objective 4 could be accomplished using a current profile that is only partially redistributed. In fact, it would be advantageous to establish a variety of plasma current profiles using current ramping as in present advanced tokamak experiments. A pulse length of $\sim 3 \tau_{\mathrm{cr}}$ would be sufficient to allow the bootstrap driven current in an advanced tokamak mode to come into equilibrium. These pulse length requirements match the capabilities of liquid nitrogen (LN) cooled copper coils, which can be designed to allow a burn time of $\sim 20$ s at full toroidal field. If advanced tokamak physics improves confinement relative to ITER design guidelines by $25 \%$ and $\beta$ by $50 \%$, then the toroidal field and plasma current can be reduced by $25 \%$ while maintaining high plasma performance (e.g., Q 10). This small reduction in the field of the FIRE copper magnet cooled to LN temperatures would allow the burn time to be increased to $\sim 40 \mathrm{~s} \sim 3 \tau_{\mathrm{cr}}$. 


\section{FIRE Device Parameters for Initial Evaluation}

The FIRE plasma configuration is an extension of the advanced tokamak programs on DIII-D and Alcator C-Mod, and is $\mathrm{a} \approx 1 / 3$ scale model of ARIES-RS, the present vision for an advanced tokamak fusion reactor. The FIRE plasma has a size and shape very similar to the previously proposed advanced tokamak (TPX), with the added capability of high performance D-T operation. The capability of FIRE to carry out long-pulse non-burning plasmas experiments will be described in a later section. FIRE will have the flexibility to incorporate new innovations as the ongoing advanced tokamak program develops them. The parameters summarized in Fig.4 were chosen as likely to achieve the FIRE mission at the lowest cost based on results of prior design studies for burning plasmas experiments (CIT and BPX) as well as recent information from the ITER-EDA and ITER-RC design activities.

\section{Capability for Alpha-Dominated Burning Plasma Experiments on FIRE}

The plasma performance of FIRE is estimated using the guidelines similar to those used to project the performance of ITER. The primary considerations are the maximum density limit, plasma energy confinement, the maximum pressure $(\beta)$ limit, the power threshold for accessing the high confinement mode (Elmy H-mode) and limitations imposed by impurities due either to alpha ash accumulation or impurities from the first wall and divertor. FIRE assumes an operating density $<0.7$ of the Greenwald density closer to those in the ITER confinement data base rather than the higher values assumed in the ITER performance projections. FIRE assumes a slightly more peaked density profile (identical to that used in the CIT and BPX projections) than ITER due to the potential for tritium pellet injection into a much smaller high-density modest temperature plasma. The FIRE projections assume the ITER98 Elmy H-mode confinement scaling relation, $\beta_{\mathrm{N}} \leq 2.5$ and the same H-mode power threshold formula as ITER. FIRE takes credit for lower impurity fractions $(\sim 3 \% \mathrm{Be})$ characteristic of high-density tokamak plasmas. In particular, FIRE assumes no significant high-Z impurities in the plasma core.

It is important to note that while these guidelines are quite useful for estimating the $n \tau_{\mathrm{E}} \mathrm{T}$ performance of existing tokamaks to within 30\%, the guidelines are mainly empirical with a modest amount of theoretical understanding and can not accurately predict the performance of a Next Step Burning Plasma experiment. An affordable flexible experiment with a performance capability about midway between today's tokamaks and a fusion reactor is needed to benchmark physics understanding and to serve as a Stepping Stone to a reactor.

The strategy of FIRE is to minimize the extrapolation in $\tau_{\mathrm{E}}$, the most uncertain quantity. The fusion gain is maximized by maximizing $\mathrm{n} \tau_{\mathrm{E}}$ at a plasma temperature of $\sim 10 \mathrm{keV}$. Analysis of the power balance in the plasma, first done by J.D. Lawson, shows that $\mathrm{n} \tau_{\mathrm{E}}$ values of $\sim 4 \mathrm{x} 10^{20} \mathrm{~m}^{-3} \mathrm{~s}$ are required to achieve $\mathrm{Q}$ values $\sim 10$ for a D-T plasma with modest impurity contamination and typical profiles. The compact high field tokamaks (IGNITOR and FIRE) reduce the requirement on $\tau_{\mathrm{E}}$ by operating at densities almost an order of magnitude higher than larger lower-field devices such as ITER. Operating in the high-density regime $n_{\mathrm{e}}(0)=6.75 \times 10^{20} \mathrm{~m}^{-3}$ is a straightforward matter since Alcator C-Mod has already operated up to $\sim 10^{21} \mathrm{~m}^{-3}$. For $\mathrm{Q}=10$, FIRE requires an energy confinement time, $\tau_{\mathrm{E}}$, of only $\sim 0.6 \mathrm{~s}$, which has been achieved in existing tokamaks such as JET, compared to the $\sim 2.5 \mathrm{~s}$ required in the reduced size ITER or the $6 \mathrm{~s}$ required for ignition in ITER. The dimensionless confinement time, $\mathrm{B} \tau_{\mathrm{E}}$, is useful to quantify the extrapolation required in plasma energy confinement from present experiments to potential Next Step Options (Table II) for burning plasmas.

Table II. Extrapolation of dimensionless energy confinement time for potential Next Step Options relative to JET.

\begin{tabular}{|c|c|c|c|c|}
\hline & JET & FIRE $(Q=10)$ & ITER-RC $(Q=10)$ & ITER-EDA $(Q=\infty)$ \\
\hline $\mathrm{B} \tau_{\mathrm{E}}(\mathrm{T}-\mathrm{s})$ & 3 & 6 & 14 & 34 \\
\hline
\end{tabular}


The extrapolation to $\mathrm{Q} \sim 10$ conditions in FIRE is a factor of two beyond JET, while ITER-RC requires a factor of four extrapolation, and ITER-EDA required an extrapolation of $\sim 11$ to achieve the objective of ignition.

The plasma parameters for a nominal FIRE operating point were calculated using a zero dimensional model. The alpha heating fractions for FIRE and ITER-RC are illustrated in Fig.5 under the assumptions of modestly peaked density profiles (triangles) and flat density profiles (crosses). The initial design point selected for FIRE satisfies all of the design guidelines needed to access the alpha dominated range with $\mathrm{P}_{\alpha} / \mathrm{P}_{\text {heat }} \geq 0.5(\mathrm{Q} \geq 5)$. This represents more than an order of magnitude advance beyond the capability of TFTR/JET to study alpha driven physics, and would provide a checkpoint more than half way to the alpha heating fraction $\mathrm{P}_{\alpha} / \mathrm{P}_{\text {heat }} \geq 0.8$ required in a fusion reactor.

The technical basis for a compact high-field tokamak like FIRE has improved markedly since the CIT $(\mathrm{R}=2.14 \mathrm{~m})$ and BPX $(\mathrm{R}=2.59 \mathrm{~m})$ studies of 1989-91. Tokamak experiments (1989 1999) have led to the development of a new scaling relation (e.g., ITER-98H) which predicts 1.3 times higher confinement than the 1989 CIT design assumption. Alcator C-Mod, which can be considered as a prototype of FIRE, has come into operation and demonstrated:

- Confinement of 1.4 times the 1989 CIT design assumptions, 15\% higher than the ITER - 98H scaling.

- High power density ICRF heating of high density shaped plasmas with a divertor.

- Detached divertor operation at high power density.

In addition, D-T experiments on TFTR and JET have shown that tritium can be handled safely in a laboratory fusion experiment. The D-T plasmas behaved roughly as expected with slight improvements in confinement for the very weak alpha heating conditions available. The behavior of the energetic alpha particles was in agreement with theoretical expectations.

The performance projections indicate that FIRE is also capable of exploring strongly alphadominated regimes with $\mathrm{P}_{\alpha} / \mathrm{P}_{\text {heat }} \geq 0.66(\mathrm{Q}=10$ to 30$)$ if the relatively higher performance (H98 $=1.2$ ) of the smaller compact high field tokamak, Alcator C-Mod, or the top end of the JET confinement results are obtained at burning plasma conditions in FIRE. During the next several years the ongoing world wide advanced tokamak program is expected to provide additional improvements of at least $\sim 25 \%$ in confinement and $50 \%$ in $\beta$. This capability would allow FIRE to explore "ignited" plasma conditions with $\mathrm{P}_{\alpha} / \mathrm{P}_{\text {heat }} \geq 0.8$ (Q up to 30) at reduced fields and longer pulses comparable to the plasma current redistribution time.

Success with FIRE would establish the physics basis for an advanced toroidal MFE reactor, namely a smaller and cheaper system obtained by introducing modest advanced features to make the tokamak, the most advanced MFE system to date, even more attractive as a fusion reactor concept.

\section{Capability for Long Pulse Advanced Tokamak Experiments in FIRE}

The development, exploration and detailed understanding of high-confinement, high-fusion powerdensity and steady-state (high-duty-cycle) plasmas are needed to provide the basis for economically attractive applications of fusion power. FIRE has significant capability to address the "steadystate" advanced toroidal configuration initiative of the Multi-Machine Strategy using non-burning deuterium plasmas, the second Stepping Stone of Fig.1.

The issues to be addressed include:

3. Optimization of confinement and $\beta$ using current and pressure profile control

2. Integration of high performance plasma with long pulse particle and power exhaust

3. Development of controls and techniques to avoid or mitigate the effects of disruptions 
The objective of FIRE would be to extend these studies beyond the plasma performance and duration accessible in present tokamaks to values closer to those for a fusion plasma. The physics of a fusion plasma is characterized by the dimensionless parameters, $\rho^{*}$ (normalized gyro-radius), $v^{*}$ (normalized collision frequency) and $\beta$. Existing tokamaks are able to replicate the $v^{*}$ and $\beta$ for a fusion reactor plasma but not simultaneously $\rho^{*}$, which requires a plasma with a larger $\mathrm{Ba}$. The important time scale for exploring advanced toroidal physics is $\tau_{\mathrm{cr}}$ the time for the plasma current profile to redistribute after a disturbance. The current redistribution time is often called the plasma current skin time. A plasma duration of $\sim 3 \tau_{\mathrm{cr}}$ would be sufficient to allow the current profile to relax to within $5 \%$ of the steady-state current profile, and would be sufficiently long to address long pulse advanced tokamak physics.

The flat top of the magnetic field in FIRE increases rapidly as the magnetic field is reduced reaching $43 \mathrm{~s}$ at $\mathrm{B}_{\mathrm{t}}=8 \mathrm{~T}$ or $225 \mathrm{~s}$ at $\mathrm{B}_{\mathrm{t}}=4 \mathrm{~T}$. In FIRE, the skin time is typically $\sim 13 \mathrm{~s}$ at $\mathrm{Q} \sim 10$ parameters due to the small minor radius, so pulses with burn times $\sim 40$ s would provide the capability to study advanced tokamak physics.

FIRE operated at $8 \mathrm{~T}$ would be able to extend the range of long-pulse advanced tokamak physics studies a factor of two in $\rho^{*}$ beyond the capability of any existing shaped divertor tokamak or any under construction for "long-pulse" durations $\geq 3 \tau_{\mathrm{cr}}$. This capability would be within a factor of two of $\rho^{*}$ in ARIES-RS.

Table III. Comparison of Long Pulse Advanced Tokamak Experiments. ( $\beta / \nu^{*}=$ constant)

\begin{tabular}{|l|c|c|c|c|c|c|c|}
\hline & DIII-D & JET & KSTAR & TPX & FIRE & ITER-RC & ARIES-RS \\
\hline $\mathrm{B}(\mathrm{T})$ & 2.2 & 2 & 3.5 & 4 & 8 & 5.5 & 8 \\
\hline $\mathrm{N} \rho \dagger=1 / \rho^{*}$ & 0.25 & 0.27 & 0.25 & 0.3 & 0.45 & 1.05 & 1.0 \\
\hline Duration/ $\tau$ & 1.7 & 4 & $7-100$ & $>100$ & $\sim 3$ & $2.2-20$ & $\infty$ \\
\hline
\end{tabular}

$\dagger$ normalized to $1 / \rho^{*}$ for ARIES-RS

\section{Integration of Long-Pulse Advanced Tokamak Physics and Alpha-Dominated Burning Plasmas, the grand challenge for Advanced Toroidal Configurations, can be explored on FIRE.}

It is anticipated that over the next several years the ongoing world-wide advanced tokamak program (total funding $\sim \$ 1.2 \mathrm{~B}$ ) will develop the advanced tokamak tools and techniques to improve confinement (e.g., H98) by $20 \%$ and $\beta_{\mathrm{N}}$ by at least 50\%. Most importantly, an experiment, such as FIRE, could then exploit these techniques to extend the range of burning plasma experiments and begin to address the critical issue of integrating of advanced tokamak physics with alpha dominated plasmas, the third Stepping Stone in Fig. 1. This is the most expeditious and least costly pathway for developing the advanced tokamak, and assessing its potential as an attractive fusion reactor.

\section{Fusion Technology and Materials Development and Experience from FIRE}

FIRE could also make significant contributions to the Fusion Technology and Materials Initiative of the Modular Strategy. FIRE would produce reactor-like fusion power density and neutron wall loading, but neither FIRE nor ITER-RC could produce the neutron fluence needed to provide data on neutron damage to structural materials (Table IV). Nonetheless, some information could be obtained from tests involving blanket modules.

Table IV. Fusion neutron parameters for Next Step Options compared to reactor parameters.

\begin{tabular}{|c|c|c|c|}
\hline & FIRE & ITER-RC & ARIES-RS \\
\hline Fusion Power Density $\left(\mathrm{MWm}^{-3}\right)$ & 10 & 0.5 & 6 \\
\hline Neutron Wall Loading $\left(\mathrm{MWm}^{-2}\right)$ & 3 & 0.6 & 4 \\
\hline 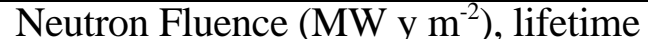 & $<0.01$ & $\sim 1$ & 120 \\
\hline
\end{tabular}


The lifetime neutron production in FIRE will be limited to $5 \mathrm{TJ}\left(3 \times 10^{-3} \mathrm{MW}-\mathrm{y} \mathrm{m}^{-2}\right)$ to reduce damage to insulators $\left(<10^{10} \mathrm{Rad}\right)$ in the toroidal field coil. The development of more radiation resistant insulators $\left(\sim 10^{12} \mathrm{Rad}\right)$ is being investigated. Consideration will also be given to the use of low activation materials in various components to gain experience for the follow-on Advanced Fusion Integration Facility. Remote handling will be implemented to maintain and replace components inside the vacuum vessel. Shielding inside the double walled vacuum vessel would allow hands-on work in the experimental areas outside the TF magnets.

\section{Plasma Technology Development from FIRE}

High speed tritium pellet injectors with vertical injection paths inside the magnetic axis and lower speed pellets guided toward the inboard mid-plane will be implemented to improve plasma performance and to reduce tritium inventory. FIRE will also develop and test materials for plasma facing components ( $\mathrm{W}$ and $\mathrm{Be}$ ) that are compatible with the fusion reactor requirement of low tritium retention. A fast low inventory tritium re-circulating system will be developed to minimize on-site tritium inventory.

The combination of high magnetic field and advanced tokamak plasma regimes could lead to very attractive fusion reactor concept with high fusion power density. This emphasizes the importance of developing the full capability of high temperature superconductors with high critical fields and high strength structural materials for fusion applications.

The plasma facing components (PFC) must withstand very high power densities while minimizing tritium retention. This is a generic problem for all MFE reactors. For the FIRE design, carbon is excluded as a PFC material due to the high retention of tritium experienced in TFTR and JET. While schemes might be developed to mitigate the carbon tritium retention issue in FIRE, the mission of FIRE is to develop a material that would also be compatible with the requirements of a high duty cycle reactor. The leading candidate for the first wall material is Be tiles in the main chamber and Be coating on copper backing plates for stabilizing plates. The power density on the outer divertor plate without peaking factors or the effects of elms is estimated to be $\sim 25 \mathrm{MWm}^{-2}$ without a detached or radiative divertor. Tungsten rods mounted on $\mathrm{Cu}$ backing plates capable of withstanding $25 \mathrm{MWm}^{-2}$ have been developed for ITER and are the leading candidate for the FIRE divertor plates. Plasma modeling is underway to ensure detached or radiative divertor operation so that there will be some margin to accommodate the effects on peaking, elms and disruptions. For the $20 \mathrm{~s}$ pulses at $200 \mathrm{MW}$ of fusion power the outer divertor plates will be actively cooled during the pulse while the inner plates and first wall tiles are cooled between pulses. Longer pulses needed to study advanced modes will require additional active cooling of the divertor and first wall which will be the subject on ongoing engineering analysis.

\section{Cost and Schedule Considerations for a Next Step Burning Plasma Experiment.}

Cost estimates have not been done for FIRE except to note comparisons with comparably sized devices, CIT $(\mathrm{R}=2.14 \mathrm{~m}, 11 \mathrm{~T})$ and BPX-AT $(\mathrm{R}=2.0 \mathrm{~m}, 10 \mathrm{~T})$, which had costs estimated to be in the range of $\$ 700 \mathrm{M}$ for experiments located at the TFTR site with about $\$ 200 \mathrm{M}$ of site credits. Some of the physical parameters that drive the costs are toroidal field magnet energy, plasma volume and plasma surface area are compared for an existing large tokamak and for potential Next Step Options in the following Table V.

The schedule to attain an MFE burning plasma test bed is an important consideration in the strategy and planning of the MFE program. Plans for the ITER-EDA required 15 years after the initiation of construction before D-T operation would be initiated; a 12 year construction period followed by three years of hydrogen and deuterium operation before D-T experiments. A similar time period would be required for ITER-RC. If ITER-RC construction were initiated in 2002, then D-T operation would begin $\sim 2017$. 
Table V. Comparison of some important cost drivers for burning plasma devices.

\begin{tabular}{|l|c|c|c|c|c|c|c|}
\hline & JET & IGNITOR & FIRE & CIT & BPX & $\begin{array}{c}\text { ITER- } \\
\text { RC }\end{array}$ & $\begin{array}{c}\text { ITER- } \\
\text { EDA }\end{array}$ \\
\hline TF Coil Technology & $\begin{array}{c}\mathrm{H}_{2} 0 \\
\mathrm{Cu}\end{array}$ & $\begin{array}{c}30^{\circ} \mathrm{K} \\
\mathrm{Cu}\end{array}$ & $\begin{array}{c}\mathrm{LN} \\
\mathrm{BeCu}\end{array}$ & $\begin{array}{c}\mathrm{LN} \\
\mathrm{BeCu}\end{array}$ & $\begin{array}{c}\mathrm{LN} \\
\mathrm{BeCu}\end{array}$ & $\mathrm{Nb} \mathrm{Sn}$ & $\mathrm{Nb}_{3} \mathrm{Sn}$ \\
\hline TF Magnet Energy $(\mathrm{GJ})$ & 1.6 & 4 & 4 & 8 & 8 & 41 & 100 \\
\hline Plasma Volume $\left(\mathrm{m}^{3}\right)$ & 85 & 11 & 18 & 35 & 65 & 740 & 2000 \\
\hline Plasma Surface $\left(\mathrm{m}^{2}\right)$ & 147 & 36 & 60 & 85 & 130 & 640 & 1200 \\
\hline Cost Range $(\$ B)$ & $\sim 0.5$ & $\sim 0.5$ & $<1$ & $\sim 0.6$ & $\sim 1.5$ & $\sim 5$ & $\sim 10$ \\
& FY84 & & & FY89 & FY92 & & \\
\hline
\end{tabular}

A small compact high field tokamak like IGNITOR or FIRE is expected to have a construction period of $\sim 7$ years followed by 3 years of hydrogen and deuterium operation based on previous schedule estimates for CIT and BPX. If construction were initiated on IGNITOR or FIRE in 2002 , then D-T operation could begin in $\sim 2012$, which is $\sim 5$ years later than the initiation of similar inertial fusion burning plasma experiments using NIF.

\section{A New Paradigm for the Magnetic Fusion Energy Roadmap}

The FIRE study indicates that a major fraction of the burning plasma physics, advanced tokamak physics, and integration of burning plasma physics with advanced tokamak physics issues could be addressed by operating a FIRE-like facility operating in different modes. This would dramatically reduce the cost and shorten the time required for the Advanced Tokamak Development Path. The Next Steps in an international Multi-Machine Strategy beyond the existing programs and facilities could include a compact high field tokamak(s) of the FIRE class, a superconducting high performance advanced tokamak of the KSTAR/TPX class and specialized fusion technology facilities to address the first wall problem for MFE. The fusion technology facilities could include intense neutron sources for materials damage studies and facilities to investigate the viability of renewable first wall concepts such as liquid surfaces facing the plasma. These facilities could provide the data needed for an Assessment of Magnetic Fusion milestone in $~ 2015$, which if successful would lead to the construction of an Advanced Fusion Integration Facility, a DEMO for MFE, with characteristics similar to those of an attractive MFE reactor. In this scenario a commercial fusion power plant could be operating midway through the $21^{\text {st }}$ century.

\section{Summary}

Exploration, understanding and optimization of alpha-dominated plasmas is a critical issue for all approaches to fusion. The tokamak is the most cost-effective vehicle to investigate alphadominated plasma physics, and its coupling to advanced toroidal physics for MFE. The performance of a burning plasma depends sensitively on the details of confinement, $\beta$-limits, density limits and edge plasma conditions. This uncertainty can only be reduced by studying actual alpha-dominated plasmas in the laboratory in conjunction with other advanced toroidal experiments and improved numerical simulations. The compact high field tokamak offers the possibility of addressing the important alpha-dominated plasma issues, many of the long pulse advanced tokamak issues and beginning the integration of alpha-dominated plasmas with advanced toroidal physics in a $\sim \$ 1 \mathrm{~B}$ class facility. Additional information can be found at http://fire.pppl.gov.

\section{Acknowledgements}

This work was performed as part of the Next Step Option Study carried out within the Virtual Laboratory for Technology and was supported by the U. S. Department of Energy under Contract DE-AC02-76-CHO3073. 


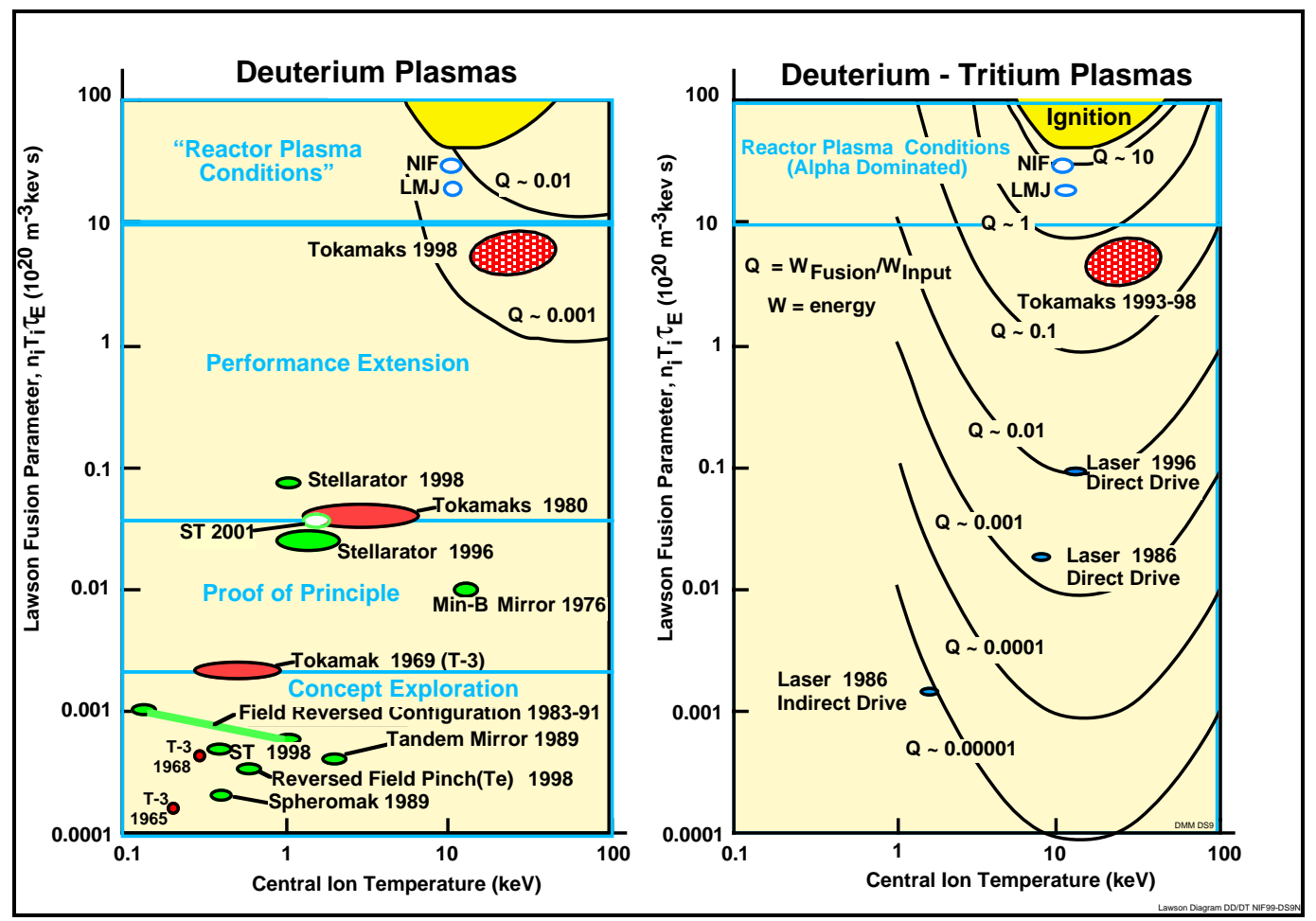

Fig. 1. The Lawson diagram showing the present status of laboratory experiments in magnetic and inertial fusion.

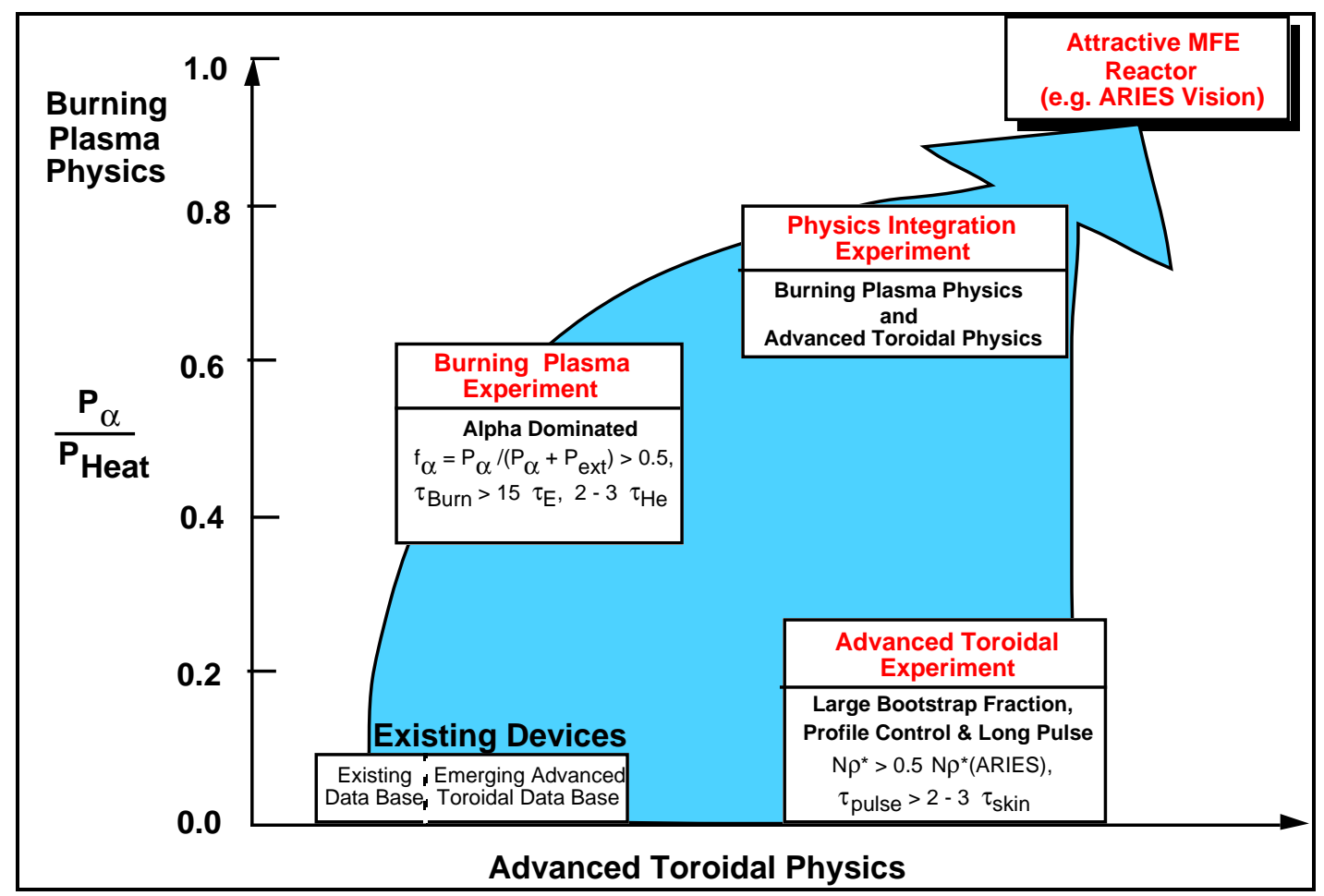

Fig. 2. The stepping stone approach for developing the science foundation of an attractive MFE system. The old paradigm was to utilize a different facility for each of the stepping stones. 


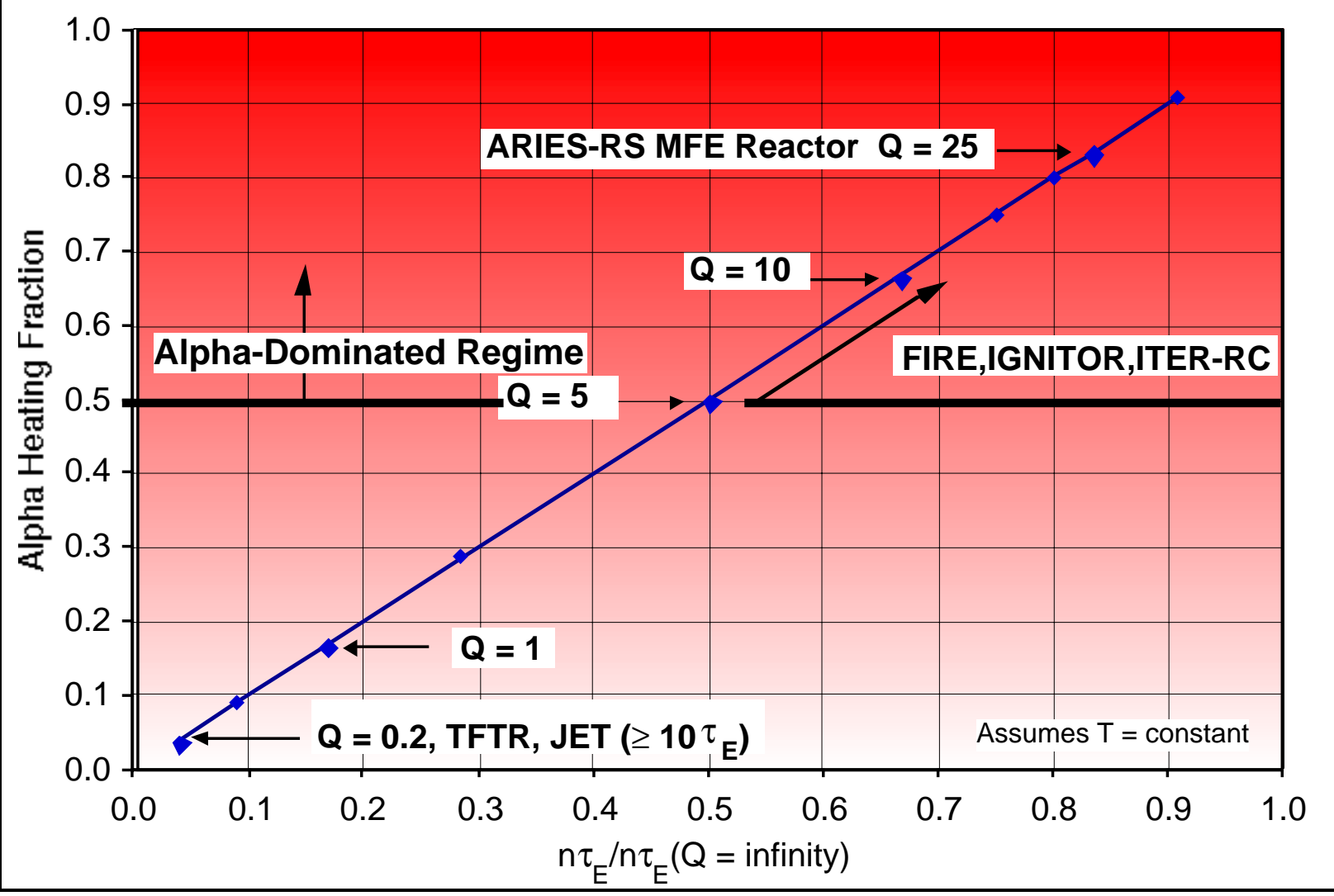

Fig. 3. Fraction of Alpha heating versus $n \tau_{\mathrm{E}} / \mathrm{n} \tau_{\mathrm{E}}(\mathrm{Q}=\infty)$ illustrating the alpha-dominated regime.

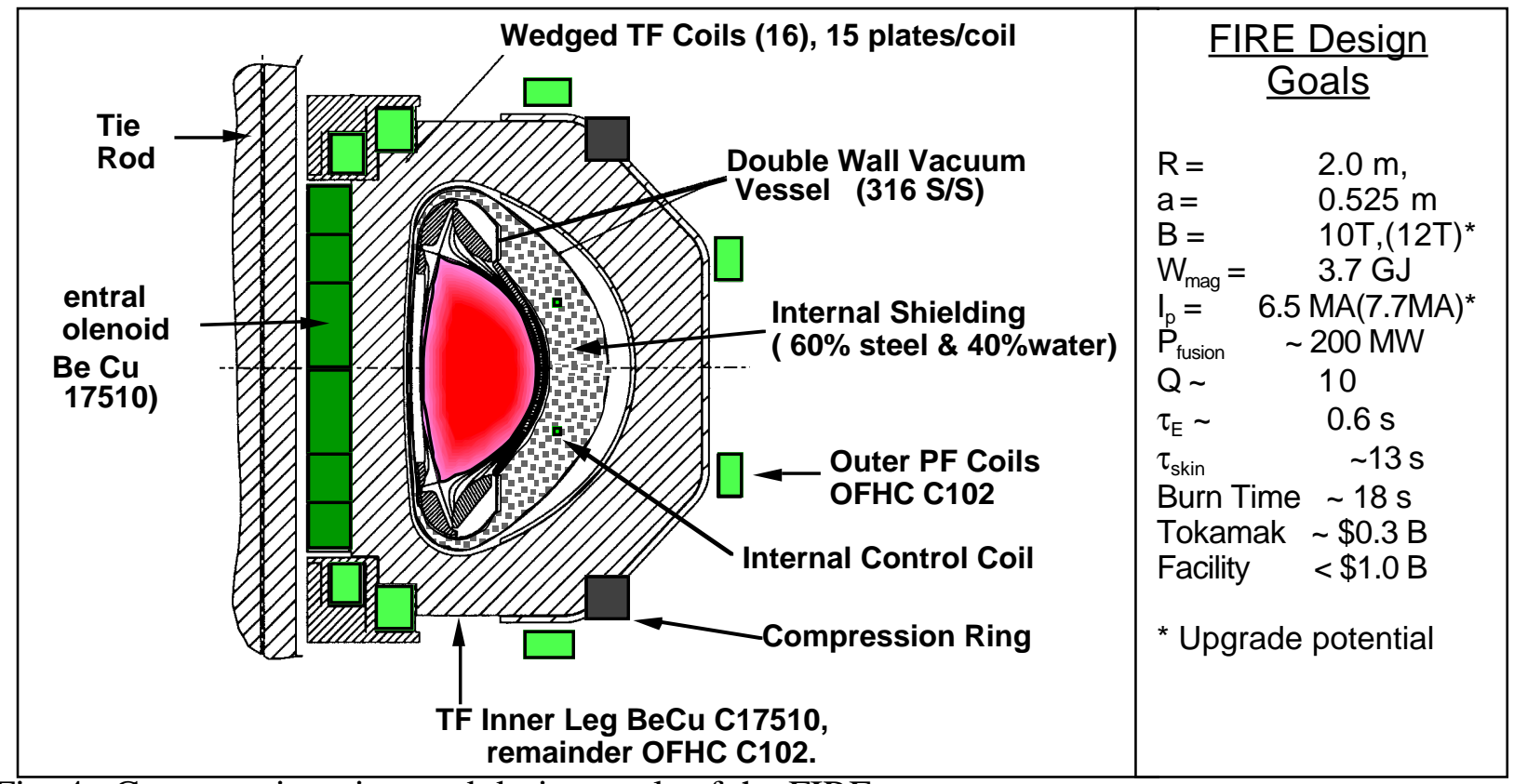

Fig. 4. Cross-section view and design goals of the FIRE. 


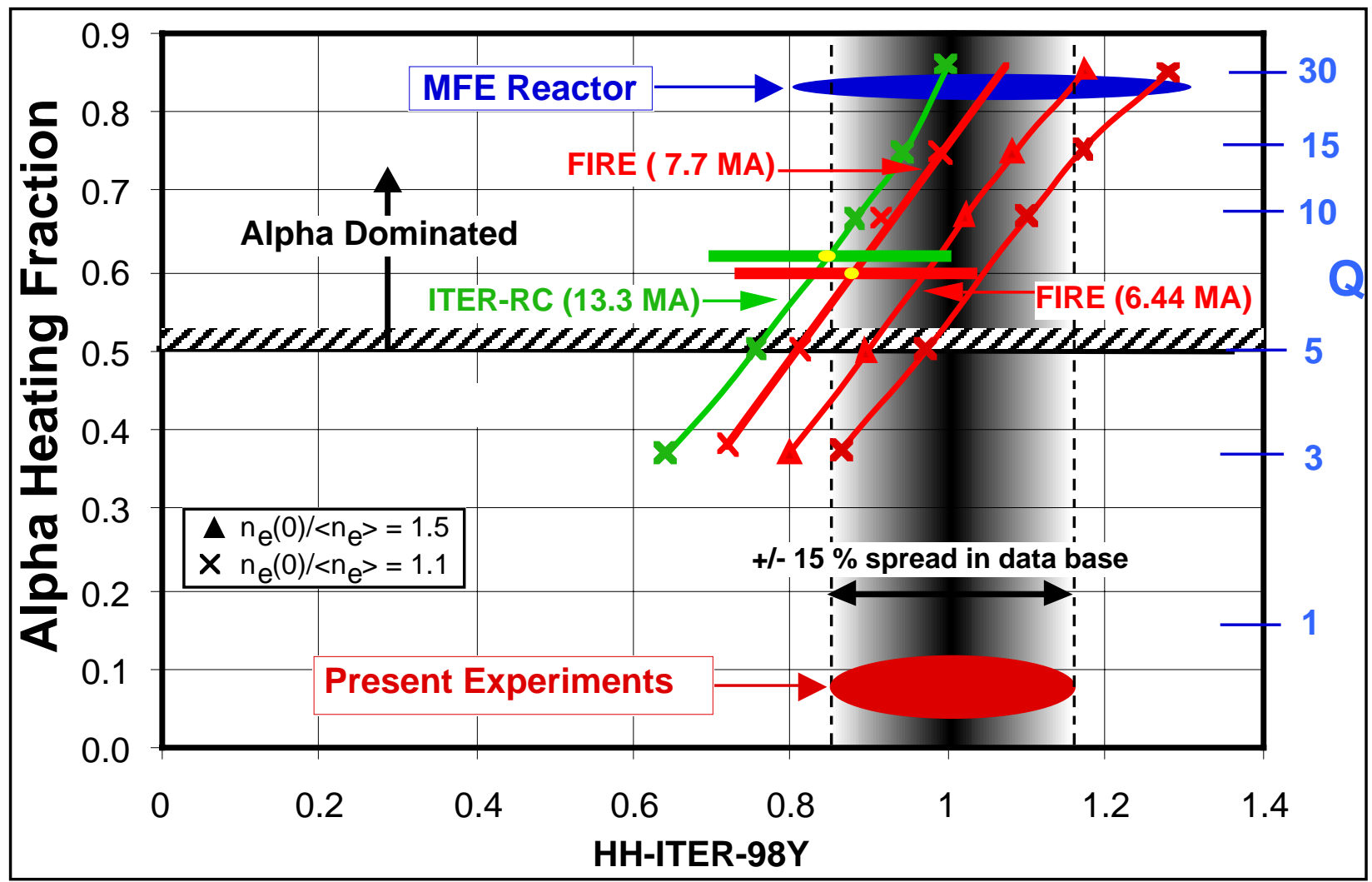

Fig. 5. Performance of FIRE and ITER-RC versus H-mode multiplier. $\mathrm{HH}=1.0$ is the center of present tokamak H-mode data base (ITER DB3). The triangles are for slightly peaked density profiles, $\alpha_{\mathrm{n}}=0.5$. The MFE reactor points are for ARIES-RS at densities ranging from 1.0 to 1.8 times the Greenwald density. 
The Princeton Plasma Physics Laboratory is operated by Princeton University under contract with the U.S. Department of Energy.

\author{
Information Services \\ Princeton Plasma Physics Laboratory \\ P.O. Box 451 \\ Princeton, NJ 08543
}

Phone: 609-243-2750

Fax: 609-243-2751

e-mail: pppl_info@pppl.gov

Internet Address: http://www.pppl.gov 